\title{
Influence of Surface Roughness on Superhydrophobicity
}

\author{
C. Yang, ${ }^{1,2}$ U. Tartaglino, ${ }^{1,3}$ and B. N. J. Persson ${ }^{1}$ \\ ${ }^{1}$ IFF, FZ-Jülich, 52425 Jülich, Germany \\ ${ }^{2}$ International Center for Theoretical Physics (ICTP), I-34014 Trieste, Italy \\ ${ }^{3}$ Democritos National Simulation Center, Via Beirut 2, 34014 Trieste, Italy \\ (Received 1 April 2006; revised manuscript received 26 July 2006; published 14 September 2006)

\begin{abstract}
Superhydrophobic surfaces, with a liquid contact angle $\theta$ greater than $150^{\circ}$, have important practical applications ranging from self-cleaning window glasses, paints, and fabrics to low-friction surfaces. Many biological surfaces, such as the lotus leaf, have a hierarchically structured surface roughness which is optimized for superhydrophobicity through natural selection. Here we present a molecular dynamics study of liquid droplets in contact with self-affine fractal surfaces. Our results indicate that the contact angle for nanodroplets depends strongly on the root-mean-square surface roughness amplitude but is nearly independent of the fractal dimension $D_{f}$ of the surface.
\end{abstract}

PACS numbers: $68.08 . \mathrm{Bc}$

The fascinating water repellents of many biological surfaces, in particular, plant leaves, have recently attracted great interest for fundamental research as well as practical applications [1-8]. The ability of these surfaces to make water bead off completely and thereby wash off contamination very effectively has been termed the lotus effect, although it is observed not only on the leaves of the lotus plant but also on many other plants such as strawberry, raspberry, and so on. Water repellents are very important in many industrial and biological processes, such as the prevention of the adhesion of snow, rain drops, and fog to antennas, self-cleaning windows and traffic indicators, low-friction surfaces, and cell mobility [9-11].

Most leaves that exhibit strong hydrophobicity have hierarchical surface roughness with micro- and nanostructures made of unwettable wax crystals. The roughness enhances the hydrophobic behavior, so that the water droplets on top tend to become nearly spherical. As a result, the leaves have also a self-cleaning property: The rain drops roll away, removing the contamination particles from the surface, thanks to the small adhesion energy and the small contact area between the contaminant and the rough leaf [1].

The hydrophobicity of solid surfaces is determined by both the chemical composition and the geometrical microor nanostructure of the surface [12-14]. Understanding the wetting of corrugated and porous surfaces is a problem of long-standing interest in areas ranging from textile science [15] to catalytic reaction engineering [16]. Renewed interest in this problem has been generated by the discoveries of surfaces with small scale corrugations that exhibit very large contact angles for water and other liquids - in some cases, the contact angle is close to $180^{\circ}$. Such surfaces are referred to as superhydrophobic [17].

The contact angle $\theta$ between a flat solid surface and a liquid droplet depends on the relation between the interfacial free energies per unit area: solid-liquid $\gamma_{\mathrm{sl}}$, solidvapor $\gamma_{\mathrm{sv}}$, and liquid-vapor $\gamma_{\mathrm{lv}}$. The Young equation $\gamma_{\mathrm{sl}}+$ $\gamma_{\mathrm{lv}} \cos \theta=\gamma_{\mathrm{sv}}$ results from the minimization of the total free energy of the system on a flat substrate surface. Complete wetting corresponds to $\theta=0^{\circ}$ and typically happens on solids with a high surface energy $\gamma_{\mathrm{sv}}$. Liquids on low energy surfaces tend to form droplets with a high contact angle $\theta$.

It is well known that the roughness of a hydrophobic solid (with $\theta>90^{\circ}$ on the flat substrate) enhances its hydrophobicity. If the contact angle of water on such flat solids is of the order of $100^{\circ}-120^{\circ}$, on a rough or microtextured surface it may be as high as $150^{\circ}-175^{\circ}$ $[11,18,19]$. Two distinct models have been proposed to explain this effect. The Wenzel model [20] considers the increase of contact area due to the surface roughness: This leads to an increase of the effective free energy of the solidliquid interface, making the surface more hydrophobic. The contact angle $\theta_{0}$ on the rough surface is obtained from the contact angle $\theta$ on the microscopically flat surface of the same material through this equation:

$$
\cos \theta_{0}=r \cos \theta \quad \text { (Wenzel model), }
$$

where $r=A / A_{0}$ is the ratio between the real substrate area and the nominal (or projected) area $A_{0}$.

The Cassie model [21] assumes that some air remains trapped between the drop and the cavities of the rough surface. In this case, the interface free energy $\gamma_{\mathrm{sl}}$ must be replaced by a weighted average of three interface free energies $\gamma_{\mathrm{sl}}, \gamma_{\mathrm{lv}}$, and $\gamma_{\mathrm{sv}}$, with the weights depending on the fraction $\phi$ of the area where the contact between the liquid and the solid happens. The contact angle is given by

$$
\cos \theta_{0}=-1+\phi(1+\cos \theta) \quad \text { (Cassie model). }
$$

Quere states that there exists a critical contact angle $\theta_{c}$ such that the Cassie state is favored when $\theta$ is larger than $\theta_{c}$ [22]. For a micro- or nanostructured substrate, usually the droplet stays in the Cassie state, but the Cassie state can switch (irreversibly) to the Wenzel state when the droplet is pressed against the substrate [23]. The Wenzel droplets are 
highly pinned, and the transition from the Cassie to the Wenzel state results in the loss of the antiadhesive properties generally associated with superhydrophobicity.

Many surfaces in nature, e.g., surfaces prepared by fracture (involving crack propagation), tend to be nearly self-affine fractal. Self-affine fractal surfaces have multiscale roughness, sometimes extending from the lateral size of the surface down to the atomic scale. A self-affine fractal surface has the property that, if part of the surface is magnified, with a magnification which, in general, is appropriately different in the direction perpendicular to the surface as compared to the lateral directions, the surface "looks the same" [24]; i.e., the statistical properties of the surface are invariant under this scale transformation.

The most important property of a randomly rough surface is the surface roughness power spectrum defined as [24-26]

$$
C(q)=\frac{1}{(2 \pi)^{2}} \int d^{2} x\langle h(\mathbf{x}) h(\mathbf{0})\rangle e^{i \mathbf{q} \cdot \mathbf{x}} .
$$

Here $h(\mathbf{x})$ is the surface height profile and $\langle\cdots\rangle$ stands for ensemble average. We have assumed that the statistical properties of the surface are translational invariant and isotropic so that $C(q)$ depends only on the magnitude $q=$ $|\mathbf{q}|$ of the wave vector $q$. For a self-affine surface, the power spectrum has the power-law behavior $C(q) \sim$ $q^{-2(H+1)}$, where the Hurst exponent $H$ is related to the fractal dimension $D_{f}=3-H$. Of course, for real surfaces this relation holds only in some finite wave-vector region $q_{0}<q<q_{1}$. Note that in many cases there is a roll-off wave vector $q_{0}$, below which $C(q)$ is approximately constant. The mean of the square of the roughness profile can be obtained directly from $C(q)$ using $\sigma^{2}=\left\langle h^{2}(\mathbf{x})\right\rangle=$ $\int d^{2} q C(q)$.

For self-affine fractal surfaces, $r=A / A_{0}$ is uniquely determined by the root-mean-square (rms) roughness $\sigma$ and the fractal dimension $D_{f}$. We have [27]

$$
A / A_{0}=\int_{0}^{\infty} d x\left(1+x \xi^{2}\right)^{1 / 2} e^{-x},
$$

where $\xi^{2}=\int d^{2} q q^{2} C(q)$. For the surfaces we use in our study, in Fig. 1 we show the ratio $r=A / A_{0}$ both as a function of the root-mean-square roughness $\sigma$ and as a function of Hurst exponent $H$. As expected, $A / A_{0}$ increases with increasing rms roughness and decreasing Hurst exponent $H$ (or increasing fractal dimension $D_{f}=3-H$ ). Qualitatively, when $D_{f}$ increases at fixed rms roughness, the short-wavelength roughness increases while the longwavelength roughness remains almost unchanged.

We have used molecular dynamics calculations to study the influence of surface roughness on superhydrophobicity. We have studied hydrocarbon liquid droplets on different self-affine fractal surfaces. The nanodroplet contains 2364 octane molecules $\mathrm{C}_{8} \mathrm{H}_{18}$ at $T=300 \mathrm{~K}$, which is between the melting and boiling points of octane. The
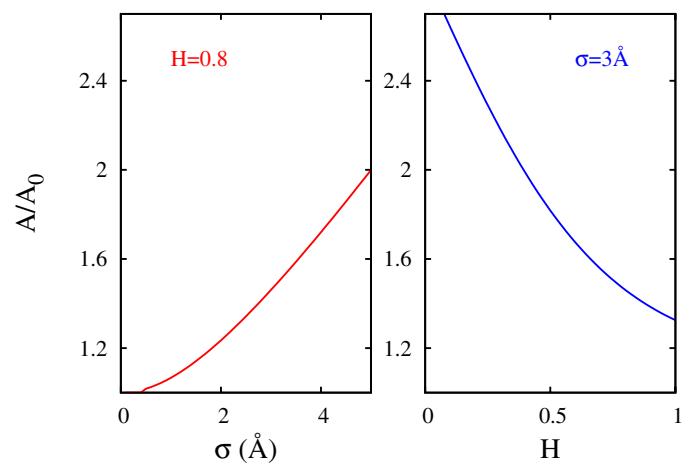

FIG. 1 (color online). The ratio $A / A_{0}$ between the actual $A$ and the nominal (or projected) $A_{0}$ surface area, as a function of the root-mean-square roughness $\sigma$ when Hurst exponent $H=0.8$ and as a function of Hurst exponent $H$ for $\sigma=3 \AA$.

fractal surfaces were generated as in Ref. [26]. Different fractal surfaces are obtained by changing the root-meansquare roughness amplitude $\sigma$ and the fractal dimension $D_{f}$. The roll-off wave vector for the rough surface is $q_{0}=$ $2 \pi / L$, with $L=38 \AA$, and the magnitude of the shortdistance cutoff wave vector $q_{1}=\pi / a$, where $a=2.53 \AA$ is the substrate lattice constant. The (noncontact) cylindrical droplet diameter is about $104 \AA$, and the size of the droplet-substrate contact area varies from $\approx 115 \AA$ [Fig. 2(a)] to $\approx 60 \AA$ [Fig. 2(c)].

The lubricant molecules are described through the optimized potential for liquid simulation [28,29]; this potential is known to provide density and viscosity of hydrocarbons close to the experimental one. We used the Lennard-Jones (L-J) interaction potential between droplet atoms and substrate atoms. The L-J parameters for a hydrophobic surface are chosen such that the Young contact angle is about $100^{\circ}$
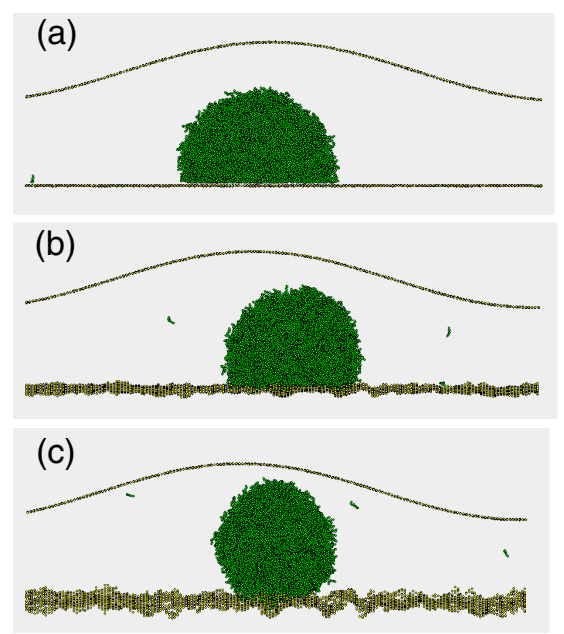

FIG. 2 (color online). Snapshots for different root-meansquare roughnesses. (a) The droplet is in contact with the flat substrate. (b) and (c) are for rough substrates with the root-meansquare amplitude $\sigma=2.3 \AA$ and $\sigma=4.8 \AA$, respectively. 


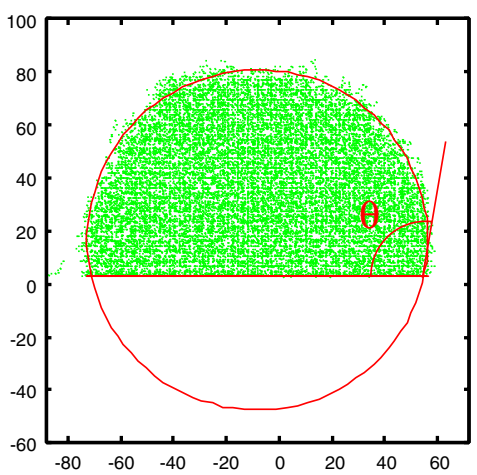

FIG. 3 (color online). Determination of the contact angle $\theta$ for the flat substrate. Side view.

when a droplet sits on the flat surface. Because of the periodic boundary condition and the size of our system, the liquid droplet forms a cylinder with the central line along the $y$ axis; see Fig. 2. We fit the density profile of the droplet to a cylinder and obtain the contact angle $\theta=103^{\circ}$ as indicated in Fig. 3 for the droplet in contact with the flat substrate.

The apparent contact angle $\theta_{0}$ as a function of the rms roughness is shown in Fig. 4 with the fractal dimension $D_{f}=2.2$. There is a strong increase in $\theta_{0}$ with increasing rms-roughness amplitude. Figure 5 shows how $\theta_{0}$ depends on the Hurst exponent $H=3-D_{f}$. Note that $\theta_{0}$ is almost independent of $H$.

Accordingly to the Wenzel equation, the apparent contact angle $\theta_{0}$ depends only on the surface roughness via the ratio $r=A / A_{0}$. Figure 1 shows that, as $H$ decreases from 1 to 0.4 (i.e., $D_{f}$ increases from 2 to 2.6 ), $A / A_{0}$ increases by $\sim 50 \%$. However, the molecular dynamics calculations show that the apparent contact angle $\theta_{0}$ is almost independent of the fractal dimension; see Fig. 5. Thus, the Wenzel equation cannot be used in the present situation. This is consistent with a visual inspection of the liquid-substrate interface, which shows that, on the rough substrates, the droplet is "riding" on the asperity top of the substrate; i.e., the droplet is in the Cassie state. In order to quantitatively verify this, we have calculated the distances $h(x, y)$ between the bottom surface of the liquid drop and the rough substrate surface in the (apparent) contact area. From the distribution $P(h)$ of these distances, we obtain the fraction $\psi$ of the (projected) surface area where contact occurs: $\psi=\int_{0}^{h_{1}} d h P(h)$, where $h_{1}$ is a cutoff distance to distinguish between contact and no-contact regions, which has to be comparable to the typical bond distance (we use $h_{1}=$ $4 \AA$ ). Note that, due to the thermal fluctuations, $\psi=\psi_{0}$ for a flat surface is less than 1 . Using the normalized $\phi=$ $\psi / \psi_{0}$, the Cassie model predicts the variation of the contact angle with $\sigma$ and $H$ given in Figs. 4 and 5 (square points).

Figure 4 shows that the apparent contact angle $\theta_{0}$ increases strongly with increasing rms-roughness amplitude,

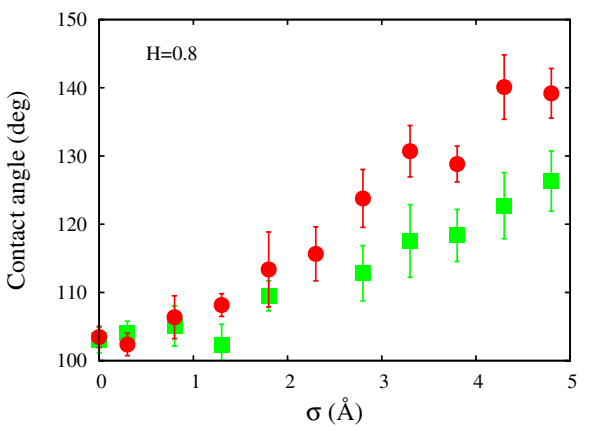

FIG. 4 (color online). The contact angle as a function of the root-mean-square roughness $\sigma$. The circle points are numerical results from the simulations, while the square points are obtained from the Cassie model [see Eq. (2)]. Each data point is an average over several snapshot configurations. The fractal dimension is $D_{f}=2.2$.

at fixed fractal dimension $D_{f}=2.2$, while it is nearly independent of the fractal dimension $D_{f}$ (see Fig. 5). Since increasing the fractal dimension at constant rmsroughness amplitude mainly increases the shortwavelength roughness, we conclude that the nanoscale wavelength roughness does not matter so much in determining the contact angle for hydrophobic surfaces, while the long-wavelength roughness plays an important role. We attribute this fact to the strong thermal fluctuations in the height (or width) $h$ of the liquid-solid interface which occur on the nanoscale even for the flat substrate surface. Note also that in our model the wall-wall interaction is long-ranged, decaying effectively as $\sim 1 / h^{3}$, so there will be a contribution to the interfacial energy also for noncontacting surfaces which, of course, is not rigorously included in the macroscopic Cassie model.

In Fig. 6, we study the hysteresis [14] in the contact angle $\theta$. In one case, a spherical droplet was attached to the substrate, leading to a decrease in the contact angle with increasing time (advancing contact angle). In the other case, the droplet was squeezed into a "pancakelike" shape by the upper wall and then released, resulting in a contact

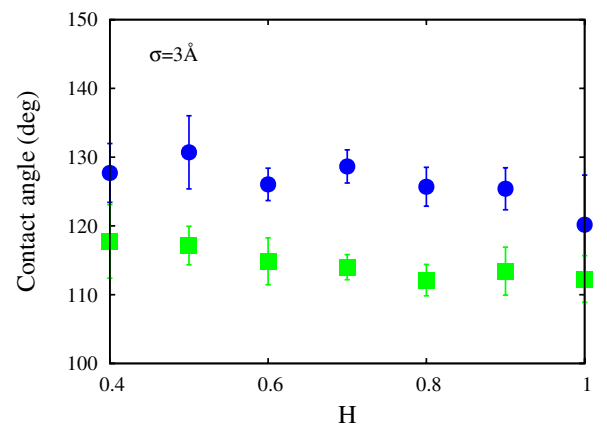

FIG. 5 (color online). The contact angle $\theta$ as a function of Hurst exponent $H$ for the rms roughness $\sigma=3 \AA$. The circles and squares have the same meaning as in Fig. 4. The fractal dimension is $D_{f}=3-H$. 


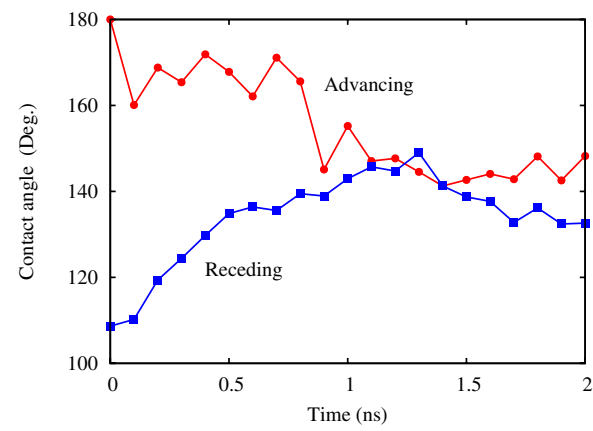

FIG. 6 (color online). The advancing (circles) and receding (squares) contact angle $\theta$ as a function of time. The thermal equilibrium contact angle has been reached after $\sim 1 \mathrm{~ns}$, irrespective of whether the initial contact angle is larger or smaller than the equilibrium angle.

angle which increases with time (receding contact angle). In both cases, the thermal equilibrium contact angle has been reached after $\sim 1 \mathrm{~ns}$. Thus, on macroscopic time scales, nanoscale roughness will not result in any hysteresis in the contact angle. This is in drastic contrast to simulation studies we have performed [30] for hydrophilic surfaces, where surface roughness results in strong pinning of the boundary line; for such surfaces, it is therefore impossible to study (advancing or receding) droplet contact angles (as observed on macroscopic time scales) using molecular dynamics.

Comparing the form of $P(h)$ for the flat and the most rough surfaces shows that the system is in the Cassie state, but on the nanoscale the difference between the Cassie state and the Wenzel state is not so large due to the thermal fluctuations. This also explains why no hysteresis is observed: The Cassie state is the free energy minimum state, and squeezing the droplet into a pancake shape does not push the system permanently into the Wenzel state, because, even if it would go into this state temporarily, the free energy barrier separating the Cassie and Wenzel states is so small that thermal fluctuations would almost instantaneously kick it back to the (free energy minimum) Cassie state.

In most practical cases, it is not possible to modify the surface roughness without simultaneously affecting the chemical nature of the surface. While this is obvious for crystalline materials, where surface roughening will result in the exposure of new lattice planes with different intrinsic surface energy, it may also hold for amorphouslike materials, where surface roughening may result in a more open atomic surface structure, with an increased fraction of (weak) unsaturated bonds. In our model study, a similar effect occurs, and some fraction of the change in the contact angle with increasing root-mean-square amplitude may be associated with this effect. However, the most important result of our study, namely, that the contact angle is determined mainly by the long-wavelength roughness, should not be affected by this fact.
To summarize, we have studied the interaction between liquid hydrocarbon nanodroplets and rough surfaces. The macroscopic contact angle $\theta_{0}$ increases with increasing root-mean-square roughness amplitude $\sigma$ of the surface, but $\theta_{0}$ is almost unchanged with increasing fractal dimension $D_{f}$. There is almost no contact angle hysteresis on the nanoscale.

This work was partly sponsored by MIUR COFIN No. 2003028141-007, MIUR COFIN No. 2004028238002, MIUR FIRB No. RBAU017S8 R004, and MIUR FIRB No. RBAU01LX5H.

[1] W. Barthlott and C. Neinhuis, Planta 202, 1 (1997).

[2] C. Neinhuis and W. Barthlott, Ann. Bot. 79, 667 (1997).

[3] A. Otten and S. Herminghaus, Langmuir 20, 2405 (2004).

[4] See www.botanik.uni-bonn.de/system/bionik for information involving surface roughness in relation to hydrophobicity and surface self-cleaning in biological systems.

[5] S. Shibuichi et al., J. Phys. Chem. 100, 19512 (1996).

[6] R. Blossey, Nat. Mater. 2, 301 (2003).

[7] N. A. Patankar, Langmuir 20, 8209 (2004).

[8] Y. T. Cheng and D. E. Rodak, Appl. Phys. Lett. 86, 144101 (2005).

[9] A. Nakajima, K. Hashimoto, and T. Watanabe, Monatsh. Chem. 132, 31 (2001).

[10] S. R. Coulson, L. Woodward, J. P. S. Badyal, S. A. Brewer, and C. Willis, J. Phys. Chem. B 104, 8836 (2000).

[11] H. Y. Erbil, A. L. Demirel, Y. Avci, and O. Mert, Science 299, 1377 (2003).

[12] R. E. Johnson and R. H. Dettre, J. Phys. Chem. 68, 1744 (1964).

[13] A. Dupuis and J. M. Yeomans, Langmuir 21, 2624 (2005).

[14] W. Chen et al., Langmuir 15, 3395 (1999).

[15] M. Suzuki, Carbon 32, 577 (1994).

[16] T. J. Barton et al., Chem. Mater. 11, 2633 (1999).

[17] J. Bico, C. Marzolin, and D. Quere, Europhys. Lett. 47, 220 (1999)

[18] S. Herminghaus, Europhys. Lett. 52, 165 (2000).

[19] A. Lafuma and D. Quere, Nat. Mater. 2, 457 (2003).

[20] R. N. Wenzel, Ind. Eng. Chem. 28, 988 (1936).

[21] A. B. D. Cassie and S. Baxter, Trans. Faraday Soc. 40, 546 (1944).

[22] D. Quere, Physica (Amsterdam) 313A, 32 (2002).

[23] G. Carbone and L. Mangialardi, Eur. Phys. J. E 16, 67 (2005).

[24] B. N. J. Persson et al., J. Phys. Condens. Matter 17, R1 (2005).

[25] P. R. Nayak, J. Lubr. Technol. 93, 398 (1971).

[26] C. Yang, U. Tartaglino, and B. N. J. Persson, Eur. Phys. J. E 19, 47 (2006).

[27] B. N. J. Persson, Eur. Phys. J. E 8, 385 (2002).

[28] W. I. Jorgensen, J. D. Madura, and C. J. Swenson, J. Am. Chem. Soc. 106, 6638 (1984).

[29] D. K. Dysthe, A. H. Fuchs, and B. Rousseau, J. Chem. Phys. 112, 7581 (2000).

[30] C. Yang, U. Tartaglino, and B. N. J. Persson (unpublished). 